\title{
Location and Orientation Based Augmented Reality Mobile Application for Enhancing Heritage Landmark History and Information
}

\author{
Savitri Galih ${ }^{*}$, Sultan Iansyah, Herdis Yudistira \\ Department of Informatics, Widyatama University, Indonesia
}

Copyright@2019 by authors, all rights reserved. Authors agree that this article remains permanently open access under the terms of the Creative Commons Attribution License 4.0 International License

\begin{abstract}
Augmented Reality technology in Mobile Device can provide better experience for people to to access and gather information at the location of cultural heritage or tourism landmark point in cultural heritage and historical tourism. Currently, the AR implementation research in cultural aspect hardly explored about the sensing algorithm of the cultural object that ignite the AR and the algorithm's complexity in detail. In this paper, we we propose geodetic location based tracking algorithm that has low computational complexity compare to the object based tracking which required heavy computational complexity for image processing algorithm. Based on the testing and implementation evaluation, our application can mark the cultural heritage or historical landmark and overlay it with the story behind the place to enhance the visitor experience.
\end{abstract}

Keywords Augmented Reality, Landmark Heritage, Android

\section{Introduction}

Nowadays mobile computing technology improvement and expansion increase significantly. It is boosted up by the advance of the special and dedicated sensors in mobile phone that is able to attain any environmental information all over us, such as camera, accelerometer, orientation, location, gyroscope, e.t.c. This development, drive the interactive technologies such as Augmented Reality (AR) to become more possible and easy to implement widely. Augmented Reality is a visualization technology that overlay text, graphics, video, sensors data and other formats on the top of camera view in mobile phone /computer or other devices.

Presently AR technology is implemented in various field, such as Military and law enforcement, vehicles, medical, tourism, architecture, industry, entertainment, education, art, weather forecasting and heritage culture to casual users. In cultural heritage and historical tourism, AR based mobile application provide a more authentic and interactive way to access and gather information at the location of cultural heritage or tourism landmark point.

Some AR research in cultural field (Museums, City landmark, e.t.c) are carried out in term of finding the increasing of visitor enhancement after applying AR [1], adding sensing system to the AR apps to adapt apps visualization with psychological state of visitor [2], proposing archetypal development framework to accommodate specific requirement of users in AR Apps for tourists sectors [3][16]. However, these research hardly explored about the sensing algorithm of the cultural object that ignite the AR and the algorithm's complexity in detail.

Another research proposed environment sensing and retrieval with comparing the real object to some dataset for tracking the object feature with relatively heavy computational complexity for image processing algorithm and required extensive storage for the datasets $[4,5,6]$.

The choice of the AR sensing algorithm is important especially for the cultural place in rural area with limited communication infrastructure since it required more light computational complexity during sensing process. In this work, we propose geodetic location based tracking algorithm that has less computational complexity than real object tracking based algorithm in $[4,5,6]$ and implement it in android mobile device.

This research objective is to develop an augmented reality mobile application that mark the cultural heritage or historical landmark and overlay it with the story behind the place to enhance the visitor experience.

\section{Augmented Reality in Mobile Computing}

Augmented Reality is defined as "an enhanced version 
of reality created by the use of technology to overlay digital information on an image of something being viewed through a device (such as a smartphone camera)" [13]. AR can be implemented in various devices, such as head-mounted displays (HMD), projector devices, and also mobile devices, since nowadays mobile device has computational ability that enables AR technology. The AR implementation in mobile devices usually comprise two main elements: the real data that sensed by the device (Camera capture, current location, current direction of device e.t.c) and the composed data used for augmentation (Additional information, image, animation e.t.c). The two elements is then complemented each other to build an AR application in a way that the composed data will overlay on the real live data. To develop the AR Application in mobile device, we need to utilize the device camera, graphics and sensors using related APIs.

In AR application development, tracking technique is a key challenge to be counted carefully. The tracking technique is selected, generally, base on the resources provided in mobile device especially the type of sensors available in the mobile devices i.e; camera, GPS, orientation sensor, accelerometer, gyroscopes e.t.c. . The several tracking techniques is discussed in the following:

a. Marker-based tracking is commonly used in limited sensor function mobile devices, since it can be simply processed and identified by camera with low computational complexity. However, this tracking technique is more suitable for indoors tracking.

b. Marker-less Tracking usually employs more complex image recognition and image processing algorithms to identify and recognize the AR targets, since it must recognize the real objects that have various shape and perspective. This condition, results in the significant increase of the system computational complexity which in turn requires higher resources (higher power, storage, computation speed etc). Thus, the technique is difficult to implement in mobile device since it has limited storage, power, etc.

c. Location Based Tracking can be used for outdoor tracking with relatively low computational complexity. However, in term of accuracy this tracking can not be guaranteed since the use of GPS base on Base Station Triangulation has wide area of tracking.

To achive more accuracy in tracking the AR target object, we use Low complex Location Based Tracking that combined with the azimuth calculation based on Orientation Sensor listener in android mobile device.

\section{System Design}

\subsection{Overview Scenario and System Architecture}

Define During the cultural heritage or historical landmark observation process, visitor will hold the mobile device point to the certain location facing to the target object. When the point of interest is displayed at the center of the camera, the augmented reality content will also displayed and superimposed upon the point of interest. In this work, the location based augmented reality Mobile Application is developed using Android Studio Tool. The main objective of this system is to recognize the point of interest (i.e the heritage landmark) and then overlay the camera view of the real-life point of interest with the augmented reality object. The overlayed AR object, therefore, is expected to enhance the visitors' interest and sensation during observing the real object.

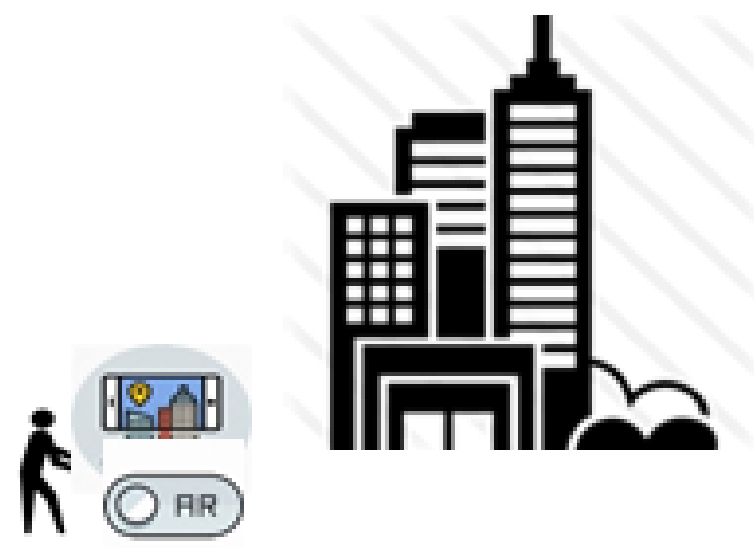

Figure 1. Augmented Reality Mobile Apps Illustration

The augmented reality content is being adjusted with the history and cultural background of the place/landmark. The challenge that are faced for developing this application is how to simply detect the point of interest and display the relevant content that can promote the information about the point of interest.

\subsection{Tracking Algorithm}

The Augmented Reality system generally exploit image processing algorithms to identify and recognize some special feature of object (point of interest) in the camera and then display the right AR feature at the real object camera image. Since the image processing algorithm require relatively heavy computation the system developed in this project used location based real object identification algorithm. The Algorithm implemented in the system is based on the geodetic Azimuth combine with the location data of the point of interest. Azimuth is defined as an angle between a point and $\mathrm{x}$-axis in the $\mathrm{x} y$ plane in spherical coordinates (q) as seen in figure 2 [12] [13].

In case of earth, we can define the geodetic azimuth as angle measured from the reference line, usually from the north. We can utilize the azimuth to identify our point of interest combining it with the location data since the azimuth only represent object direction. The right direction 
and the right location will designate the exact position of our point of interest and the right AR object can be generated and displayed in the real position through camera view.

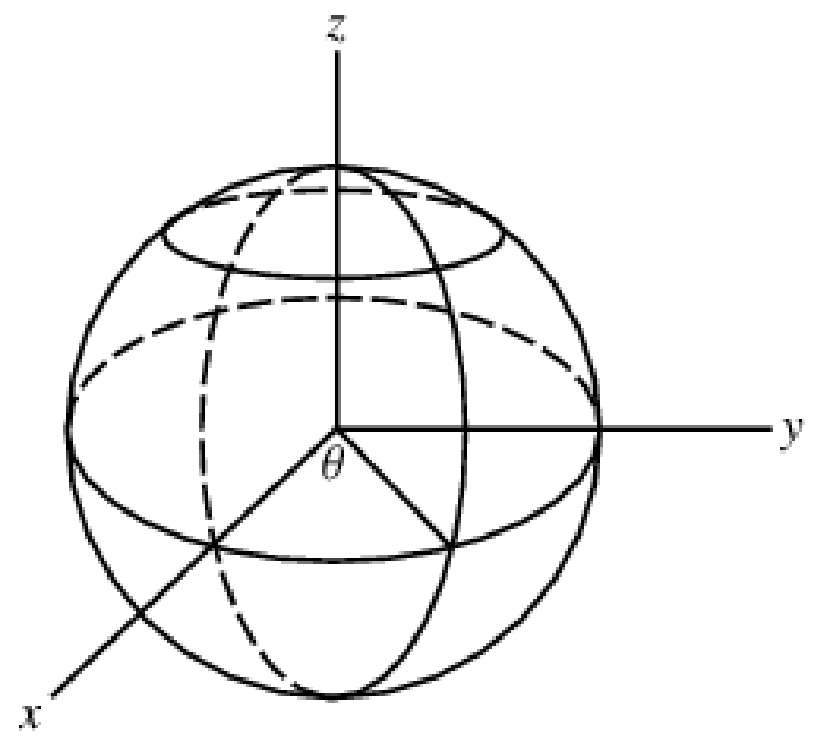

Figure 2. The Geometric Picture of Azimuth Definition

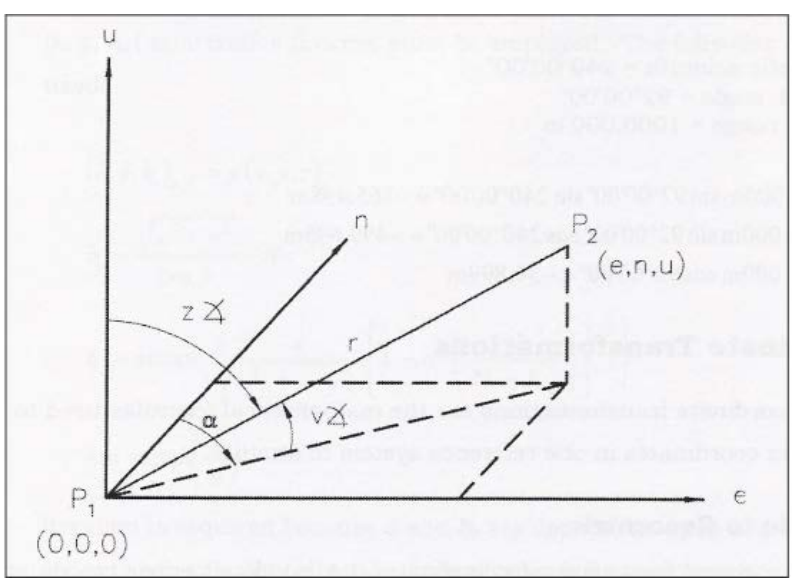

Figure 3. The location (coordinate) and geodetic azimuth of point $\mathrm{P}_{2}$

As per azimuth definition, figure 3 showed the geodetic azimuth and location of point P2 under 3 axes coordinate: e, $\mathrm{n}$ (north) and $\mathrm{u}$. The coordinate value of P2 is denote as

$$
\begin{aligned}
& e=r \cos (v \angle) \sin \alpha=r \sin (\mathrm{z} \angle) \sin \alpha \\
& n=r \cos (v \angle) \cos \alpha=r \sin (z \angle) \cos \alpha \\
& u=r \sin (v \angle)=r \cos (\mathrm{z} \angle)
\end{aligned}
$$

The geodetic azimuth of point $\mathrm{P}_{2}$ denoted as

$$
\alpha=\arctan \left(\frac{e}{n}\right)
$$

Where $\mathrm{r}=\mathrm{P} 2$ range from zero coordinates; $\mathrm{v}$ and $\mathrm{z}$ are vertical and zenith angle respectively [15]

Geodetic azimuth in equation (2) doesn't consider the earth curvature or usually called planar approximation, hence, in the real earth condition for the point of interest location that relatively far from north pole, such as Indonesia and other countries located at equator, this azimuth calculation have high distortion. Therefore, in this work we plan to implement spherical geodetic azimuth algorithm that can be more precisely recognize the point of interest to be overlayed by AR object.

The spherical approximation for calculating azimuth is chosen since it indicate error less than $1 \%$ for flattening in the order of $10^{-3}$ [16]. The spherical geometry is showed in figure 4
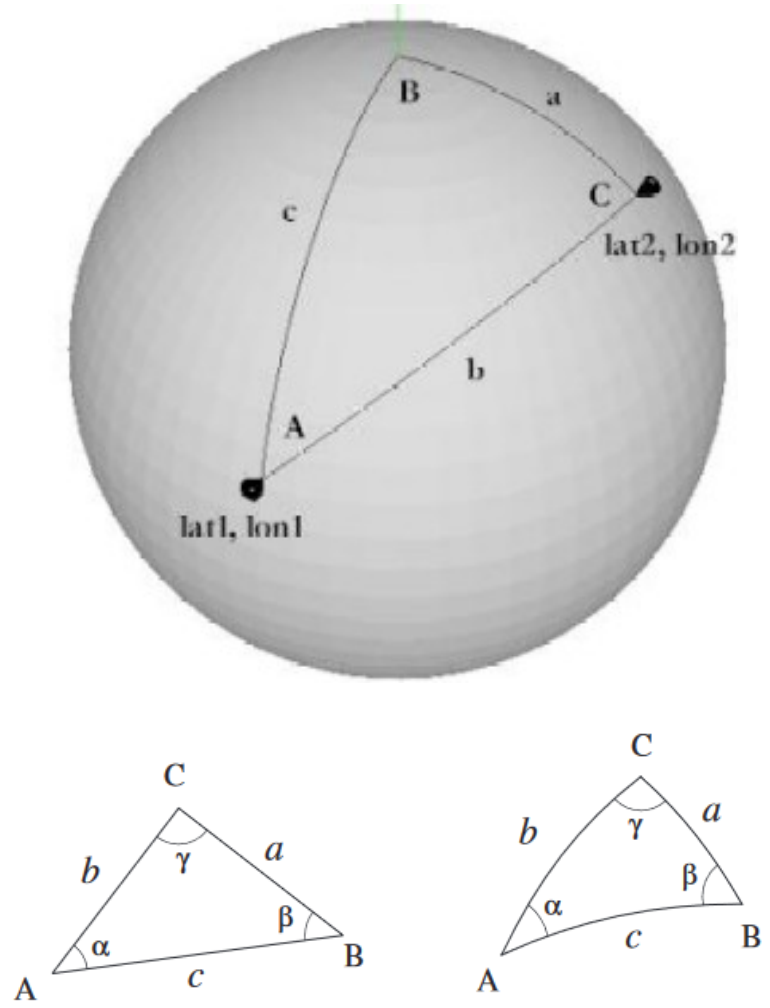

Figure 4. Spherical Geometry Azimuth

There are some differences between planar and spherical approximation despite their similarity especially in term of trigonometric relationships between angles and sides. The main different can be showed in figure $3 \mathrm{~b}$, i.e. in planar shape, the sides of triangle are in form of line, while in spherical approximation, the sides are a segment of a great circle and moreover instead of angles, a, b , g are expressed in angular units.

To calculate the azimuth (angle A12 ) in spherical model (figure 4), we need to utilize cosine and sine law. For the spherical model sine and cosine law are define as

$$
\text { Sine : } \frac{\sin \alpha}{a}=\frac{\sin \beta}{b}=\frac{\sin \gamma}{c}
$$

Cosine : $a^{2}=b^{2}+c^{2}-2 b c \cos \alpha$ 


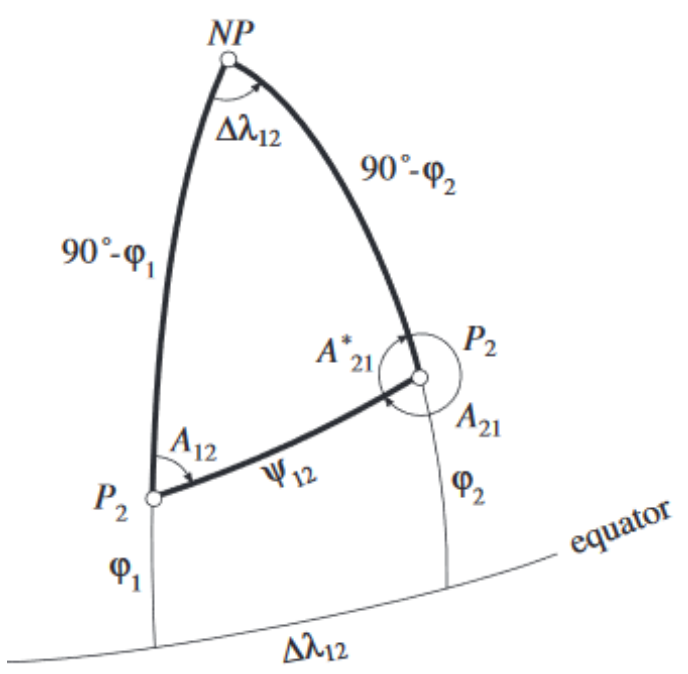

Figure 5. The Spherical triangle represent Point P2 and NP (North Pole)

To determine the Azimuth A we need to implement cosine and sine laws:

$$
\begin{aligned}
& \cos \left(90^{\circ}-\phi_{2}\right)= \\
& \quad \cos \left(90^{\circ}-\phi_{1}\right) \cos \psi_{12}+\sin \left(90^{\circ}-\phi_{1}\right) \sin \psi_{12} \cos A_{12} \\
& \Rightarrow \cos A_{12}=\frac{\sin \phi_{2}-\sin \phi_{1} \cos \psi_{12}}{\cos \phi_{1} \sin \psi_{12}}
\end{aligned}
$$

$$
\begin{aligned}
& \frac{\sin A_{12}}{\sin \left(90^{\circ}-\phi_{2}\right)}=\frac{\sin \Delta \lambda_{12} \cos \phi_{2}}{\sin \psi_{12}} \\
& \Rightarrow \sin A_{12}=\frac{\sin \Delta \lambda_{12} \cos \phi_{2}}{\sin \psi_{12}} \\
& \Rightarrow A_{12}=\arctan \frac{\sin A_{12}}{\cos A_{12}}
\end{aligned}
$$

\section{System Implementation}

The system exploits Location Service and Motion Sensor in Android Mobile Device. The location service will generate the latitude and longitude data of the current location. This data will be used to calculate the theoretical azimuth by using algorithm in section 3.b. , while the motion sensor is used to generate Real Azimuth Data. To recognize and mark the point of interest, we need to compare the theoretical and real azimuth. If both azimuth match each other, the system will invoke the augmented reality event.The augmented reality event then will overlay the AR object/model on the PoI camera view. This AR model can be a landmark Icon, historical image or a historical story to enhance visitor experience during their visit at the landmark site. Figure 6 depict the implementation of the algorithm for the AR system.

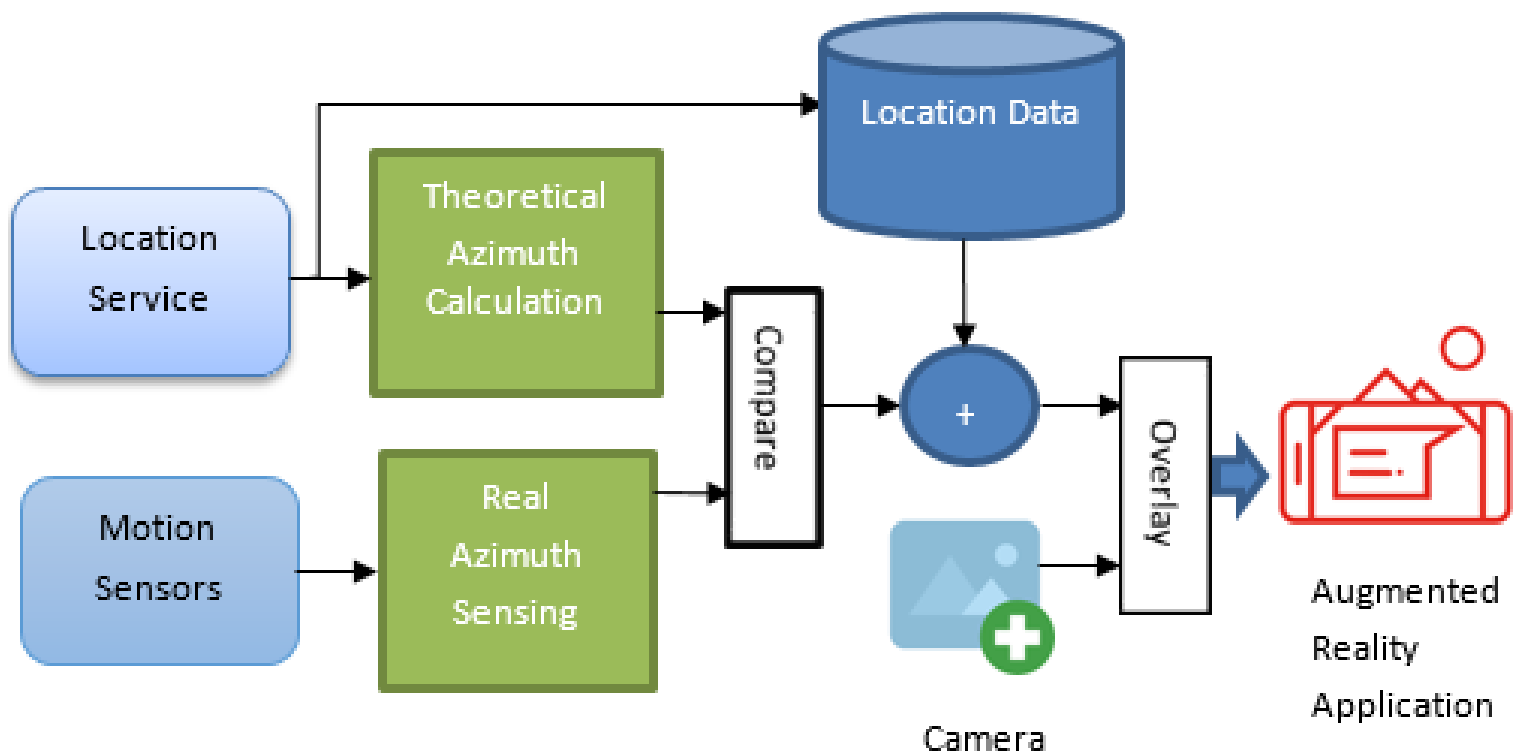

View

Figure 6. The implementation of the algorithm for the AR system 


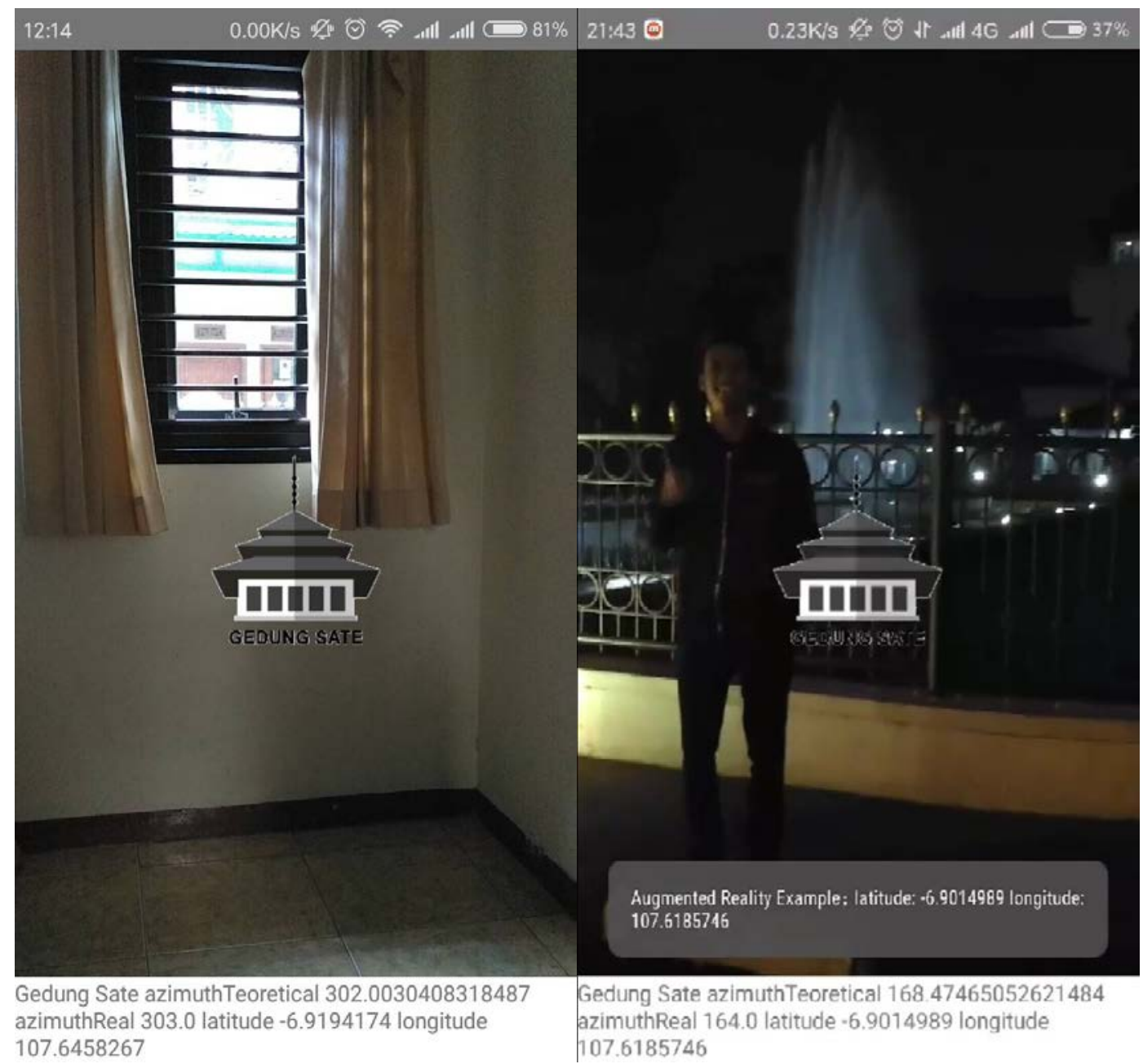

Figure 7. First Prototype AR Apps Test

The application development method is using prototyping method. The first prototype's output is simply implement geodetical data only without addition process with location data before the overlaying process. This prototye therefore display the augmented reality object when the azimuth of the place is match with the calculated azimuth even if the exact location is different. Afterward, the last prototype implement additional location data of the point of interest before overlaying the augmented reality object to improve the accuracy and the pressision of the application.

\section{Result}

By utilizing camera phone, the developed application has been tested to ensure whether the tracking algorithm employed android device's location service and motion sensors can display the Augmented Reality Model. In the case of prototype 1 the AR object is displayed not only in the exact location of the point of interest but also at the place with same azimuth data, even it has some long distance from the real Point of Interest Location (Figure 7)
The improved prototype testing result showed that the Augmented reality object show up at exact position of the point of interest, i.e. the "Gedung Sate" the famous landmark of Bandung City West Java Indonesia (figure 8). The Augmented Reality object is the historical information about Gedung Sate, that is, a battle of 7 Indonesian warrior and the allied forces during world war II.

\section{Conclusions}

The low complexity Augmented Reality mobile application that exploit geodetic information (location and orientation) of the mobile device to enhance the city landmark visitor's experienced is developed. Based on the testing process it is shown that this application can display the historical background information of Bandung Indonesia's landmark Gedung Sate at the right location.

The implication of the application is by enhancing the more location of the point of interests, the tourist visit to Indonesia specifically Bandung can be raised. Moreover it can increase the government income to support the development of Indonesia as a developing country. In the 
education field it can expand the student experience in history and geography class to increase their love to their homeland.

The future works is to increase the application's point of interest and integrate it with the history and geography curricullum in elementary and secondary school in Indonesia. Then, the evaluation in the implementation impact need to be carried out.
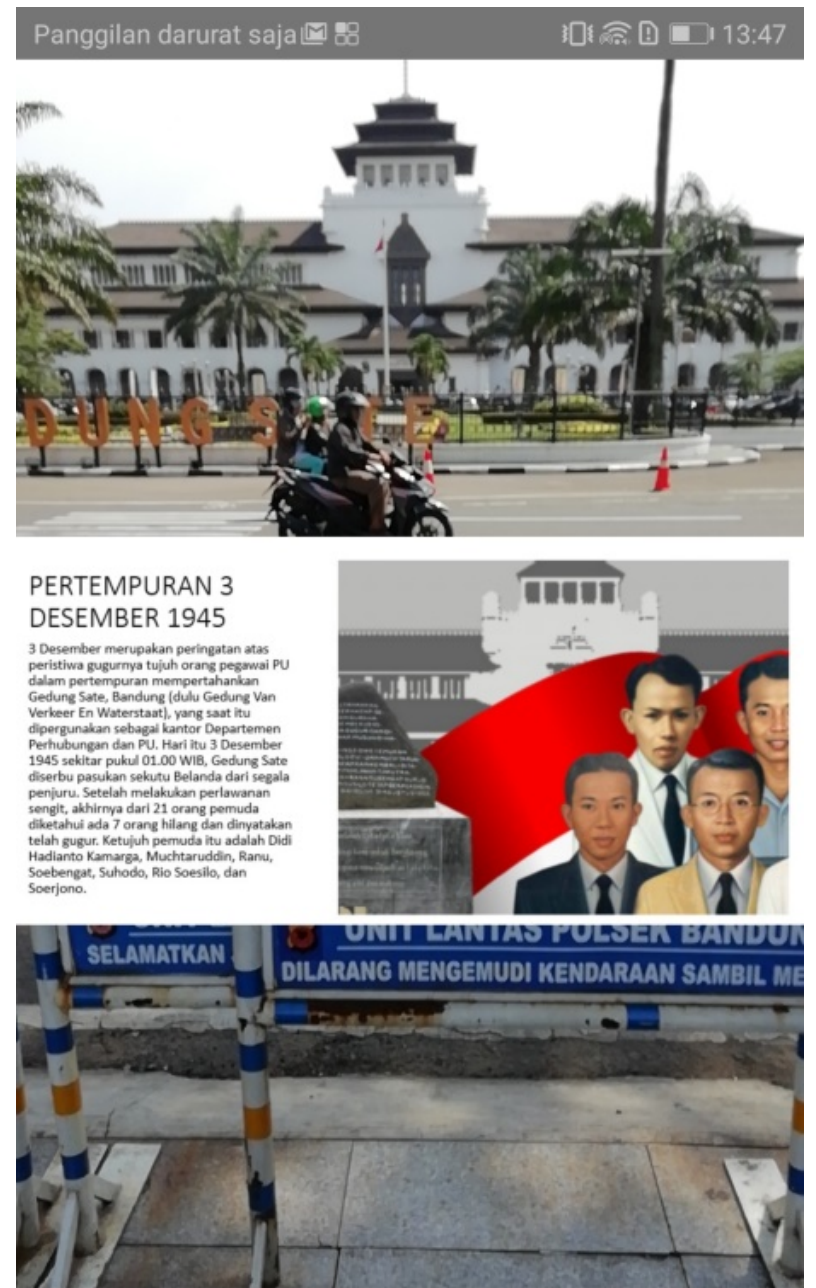

Gedung Sate azimuthTeoretical 177.67935808364646 azimuthReal 192.0 latitude -6.9014722 longitude 107.6187397

$\nwarrow \unlhd$ Tampilan layar penuh

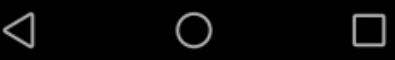

Figure 8. Last Prototype AR Apps Test

\section{REFERENCES}

[1] Chang, Y.-L., Hou, H.-T., Pan, C.-Y., Sung, Y.-T., Chang, K.-E, “Apply an Augmented Reality in a Mobile Guidance to Increase Sense of Place for Heritage Places”, Educational
Technology \& Society, 18 (2), 166-178, 2015

[2] Areti Damala, et.al, "Adaptive Augmented Reality for Cultural Heritage: ARtSENSE Project”, Proceeding of EuroMed 2012, pp. 746-755, Springer Verlag 2012

[3] Chris D. Kounavis,et.al, "Enhancing the Tourism Experience through Mobile Augmented Reality: Challenges and Prospects", International Journal of Engineering Business Management, 1 January 2012.

[4] Giovanni Murru, et.al, Practical Augmented Visualization on Handheld Devices for Cultural Heritage”, Proceeding of 21st International Conference on Computer Graphicd, Visualization and Computer Vision 2013.

[5] Suryanto, T., Haseeb, M., \& Hartani, N. H. (2018). The Correlates of Developing Green Supply Chain Management Practices: Firms Level Analysis in Malaysia. International Journal of Supply Chain Management, 7(5), 316.

[6] Gabriel Takacs, "Outdoors Augmented Reality on Mobile Phone using Loxel-Based Visual Feature Organization”, Proceedings of the 1st ACM SIGMM International Conference on Multimedia Information Retrieval, MIR 2008, Vancouver, British Columbia, Canada, October 30-31, 2008

[7] Sharma, G., \& Yadav, A. (2018). Fault Tolerance in Real Time Distributed System. Review of Computer Engineering Research, 5(2), 20-24.

[8] Giuseppe Caggianese, Pietro Neroni, Luigi Gallo, “Natural Interaction and Wearable Augmented Reality for the Enjoyment of the Cultural Heritage in Outdoor Conditions”; International Conference on Augmented and Virtual Reality AVR 2014 pp 267-282, December 2014.

[9] Eulalia Rodríguez Fino, Jorge Martín-Gutiérreza, M. Dolores Meneses Fernándezb, Enrique Armas Davarac; "Interactive Tourist Guide: Connecting Web 2.0, Augmented Reality and QR Codes”; Procedia Computer Science 25 ( 2013 ) 338 - 344;

[10] Chris D. Kounavis, Anna E. Kasimati and Efpraxia D. Zamani, "Enhancing the Tourism Experience through Mobile Augmented Reality: Challenges and Prospects", International Journal of Engineering Business Management, July 2012

[11] Han Jong-Gil, et.al., "Cultural Heritage Sites Visualization System based on Outdoor Augmented Reality”, AASRI Procedia, Volume 4, 2013, Pages 64-71

[12] Ali, A., \& Haseeb, M. (2019). Radio frequency identification (RFID) technology as a strategic tool towards higher performance of supply chain operations in textile and apparel industry of Malaysia. Uncertain Supply Chain Management, 7(2), 215-226.

[13] El-Shorbagy, M., \& Mousa, A. (2017). Chaotic particle swarm optimization for imprecise combined economic and emission dispatch problem. Review of Information Engineering and Applications, 4(1), 20-35.

[14] Wang, X., Wang, Y., Ge, J., Wu, P., \& Huang, T. (2018). Applying Building Information Modelling Plus-the Practice of Shanghai Shipping Service Complex. Review of Industrial Engineering Letters, 4(2), 21-35.

[15] Hugo Filipe Coelho Duarte Teixeira, Interactive Augmented 
Reality using mobile devices, Thesis for the degree of Master of Science in Electrical and Computer Engineering Telecommunications, Electronics and Computers Major, FACULDADE DE ENGENHARIA DA UNIVERSIDADE DO PORTO

[16] Joel Haasdyk, Choosing the best path: Global to national coordinate transformations, Coordinates February 2012

[17] Ahmed, U., Zin, M. L. M., \& Majid, A. H. A. (2016). Impact of Intention and Technology Awareness on Transport Industry's E-service: Evidence from an Emerging Economy. Journal of Industrial Distribution \& Business Vol, 7(3), 13-18.

[18] Nico Sneeuw, Geodesy and Geoinformatics part: Geodesy, Lecture Notes Institute of Geodesy University of Stuttgart October 25, 2010G.

[19] Shi, X., Dini, A., Shao, Z., Jabarullah, N.H. \& Liu, Z. (2019) Impacts of photovoltaic/ wind turbine/ microgrid turbine and energy storage system for binding model in power system, Journal of Cleaner Production, 226, 845-857. 